\title{
Structural equation modeling analysis of Iranian women's food consumption: influence of socio-demographic characteristics and the Information, Motivation, and Behavioral skills Model
}

\author{
Análise de modelagem de equações estruturais \\ do consumo alimentar de mulheres \\ iranianas: influência das características \\ sociodemográficas e do Modelo de Informação, \\ Motivação e Habilidades comportamentais
}

\author{
Maryam MOHAMMADI-NASRABADI 1 (D) 0000-0003-0340-4572 \\ Roya SADEGHI' (D) 0000-0002-4290-0950 \\ Abbas RAHIMI-FORUSHANI2 (D) 0000-0002-3052-6420 \\ Fatemeh MOHAMMADI-NASRABAD| ${ }^{3}$ (D) 0000-0001-5861-6420 \\ Davoud SHOJAEIZADE ${ }^{1}$ (D) 0000-0003-2730-4795 \\ Ali MONTAZERI ${ }^{4}$ (iD 0000-0002-5198-9539
}

\section{A B S T R A C T}

\section{Objective}

This study aimed at examining whether food consumption varies in accordance with socio-demographic and behavioral factors in the conceptual model, analyzing adult women in Tehran, Iran, for that purpose.

1 Tehran University of Medical Sciences, School of Public Health, Department of Health Education and Promotion. Qods St., n. 226, Keshavarz Blvd., 1416753955, Tehran, Iran. Correspondence to: R SADEGHI. E-mail: <sadeghir@tums.ac.ir>.

2 Tehran University of Medical Sciences, School of Public Health, Department of Epidemiology and Biostatistics. Tehran, Iran.

3 Shahid Beheshti University of Medical Sciences, National Nutrition and Food Technology Research Institute, Department of Food and Nutrition Policy and Planning Research. Tehran, Iran.

${ }^{4}$ Iranian Institute for Health Sciences Research, Health Metrics Research Center, Population Health Research Group. Tehran, Iran.

Article elaborated from the thesis by M MOHAMMADI-NASRABADI, entitled "An educational intervention to promote healthy nutritional behavior in women: design and the framework". Tehran University of Medical Sciences; 2018.

How to cite this article

Mohammadi-Nasrabadi M, Sadeghi R, Rahimi-Forushani A, Mohammadi-Nasrabadi F, Shojaeizadeh D, Montazeri A. Structural equation modeling analysis of Iranian women's food consumption: influence of socio-demographic characteristics and the Information, Motivation, and Behavioral skills Model. Rev Nutr. 2020;33:e180268. http://dx.doi. org/10.1590/1678-9865202033e180268 


\section{Methods}

This cross-sectional study was conducted with 247 women in childbearing age, who were selected through systematic cluster sampling from five regions in Tehran. Dietary assessment was done through a Food Frequency Questionnaire, and the anthropometric indices were measured. Data on socio-economic status and informationmotivation-behavioral skills model were obtained through a questionnaire. Then, subjects were stratified according to the socio-economic status and model components. Statistical analysis was done using analysis of variance and structural equation modeling.

\section{Results}

Consumption of food groups among the participating women differed based on their living region, education, occupation, household expenditure, and model components $(p<0.05)$. Intake of red and processed meat $(p<0.05)$ was at the lowest level in illiterate or low educated women. Those with lower total expenditure had higher consumption of fats and oils $(p<0.05)$. Women with higher perceived social support consumed more milk and dairy products $(p<0.05)$, fats and oils $(p<0.05)$, and less bread and cereals $(p<0.05)$. Model components including information, attitude, social support, self-efficacy, and self-regulation were the most important factors negatively affecting the consumption of unhealthy foods (red and processed meat, fats and oils, sugar, and salty foods).

\section{Conclusion}

Considering the impact of model components on women's eating behaviors, the specific integration strategies delineated for each construct of the model can be utilized to design model-based interventions targeting the promotion of healthy nutritional behavior.

Keywords: Diet. Latent class analysis. Social class. Women.

\section{RE S U M O}

\section{Objetivo}

Este estudo buscou examinar se o consumo de alimentos varia de acordo com fatores sociodemográficos e comportamentais no modelo conceitual entre mulheres adultas em Teerã, Irã.

\section{Métodos}

Este estudo transversal foi conduzido em 247 mulheres em idade reprodutiva, selecionadas por amostragem sistemática por conglomerados de cinco regiões de Teerã. A avaliação da dieta foi realizada por meio de um questionário de frequência alimentar, e os índices antropométricos foram medidos. Os dados sobre o status socioeconômico e o modelo de habilidades de informação-motivação-comportamento foram obtidos através de um questionário. Em seguida, os sujeitos foram estratificados conforme o status socioeconômico e os componentes do modelo. A análise estatística utilizou análise de variância e modelagem de equações estruturais.

\section{Resultados}

O consumo de grupos de alimentos entre as mulheres participantes diferiu de acordo com a região onde vivem, educação, ocupação, gasto familiar e componentes do modelo $(p<0,05)$. A ingestão de carne vermelha e processada $(p<0,05)$ foi mais baixa entre mulheres analfabetas ou com baixa escolaridade. Aquelas com menor gasto total apresentaram maior consumo de gorduras e óleos $(p<0,05)$. Mulheres com maior apoio social percebido consumiram mais leite e derivados $(p<0,05)$, gorduras e óleos $(p<0,05)$ e menos pão e cereais $(p<0,05)$. Os componentes do modelo, incluindo informação, atitude, apoio social, autoeficácia e autorregulação, foram os fatores mais importantes que afetaram negativamente o consumo de alimentos não saudáveis (carne vermelha e processada, gorduras e óleos, açúcar e alimentos salgados).

\section{Conclusão}

Considerando o impacto dos componentes do modelo nos comportamentos alimentares das mulheres, as estratégias de integração específicas delineadas para cada construto do modelo podem ser utilizadas para projetar intervenções baseadas em modelos visando promover um comportamento nutricional saudável.

Palavras-chave: Dieta. Análise de classe latente. Classe social. Mulheres. 


\section{NTRODUCTION}

Over the last decades, Iranian people have been undergoing a nutrition transition accompanied by major changes in their dietary patterns. The nutrition transition in Iran is occurring rapidly and against the backdrop of lack of sustained economic growth [1]. On the other hand, international research suggests that health inequalities may be partially attributable to differences in food intake across socio-economic characteristics [2].

An association between Socio-Economic Status (SES) and food intake has been recognized $[3,4]$ and inequalities in dietary intake have been associated with inequalities in health [5]. Social differences in food consumption in terms of the diets' quantity and quality have been shown in previous studies of developed countries, in which higher socio-economic levels have been associated with healthier dietary patterns (including a higher consumption of fruit and vegetables and a lower intake of fat and meat) [6-8]. These dietary differences have led to unfavorable macronutrient [9] and micronutrient [10] intake among the low-SES groups. In addition, it should be considered that, compared to single nutrient-based recommendations, food group-based recommendations could be more easily understood by people because nutrients are consumed via food [11]. The Food Frequency Questionnaire (FFQ) is a quick, practical, and efficient approach to assess subjects' habitual diets over periods of time, with lower investigator and respondent burden [12].

Although previous studies have indicated the association between socio-demographic characteristics and food group intake $[2,13]$, limited studies have simultaneously explored the direct and indirect associations of food group intake, socio-demographic factors, and the Information, Motivation, and Behavioral Skills (IMB) model using a conceptual model [14] which has been tested with Structural Equation Modeling (SEM). The Information, Motivation, and Behavioral Skills model presumes that a knowledgeable and motivated person is more inclined to develop and enact the related behavioral skills and more likely to be engaged in targeted healthy behaviors. This model has been extensively applied to predict positive health behaviors in multiple populations. In addition, there is a lack of knowledge about how SES and IMB components mediate and simultaneously influence food group consumption, especially in women living in developing countries. Information, motivation, and behavioral skills are the three constructs taken as generalizable determinants of health behaviors. Thus, the IMB model has been used as a theoretical framework for behavioral intervention studies across a variety of health behaviors [15].

The present study aimed at determining whether socio-economic differences have an effect on diet and food group consumption and testing a conceptual model of associations between sociodemographic and behavioral factors, and food intake among adult women in Tehran, Iran.

\section{METHODS}

This cross-sectional study is part of the project entitled "Educational intervention to promote healthy nutritional behavior in women of Tehran", carried out during 2016 and 2017. The Ethical Committee of Tehran University of Medical Sciences, Tehran, Iran approved the study (protocol n. 8921108011-131264). This study was also registered in Iranian Registry of Clinical Trials (IRCT2013012412255N1).

Through systematic cluster sampling, 247 women aged 20-49 years old were selected from five districts (North, South, East, West, and Center) at the Health Houses affiliated to the Municipality 
of Tehran, the capital of Iran. After getting written informed consent, the researchers filled in a 30-45 minute questionnaire for each participant through face-to-face interview. In order to collect the data, as well as anthropometric measures, the participants were asked to answer the questions regarding SES, IMB components, and food frequency.

For appropriate sample size in structural equation modeling, about 20 samples are required for each agent (latent variable). Most studies have shown that a sample size of 150 is also sufficient to conduct exploratory factor analysis. For a confirmatory factor analysis, a minimum sample size of 200 is proposed [16]. Therefore, for this study, 240 participants were included.

Data on the socio-demographic characteristics of the women including the age, educational level, and occupational status of such women and their husbands, as well as family size, expenditures, and residency were gathered through a questionnaire completed by trained interviewers. The subjects were classified into three categories (low, middle, and high) according to the tertile of total family expenditure.

The height, weight, and Waist Circumference (WC) of the studied women were measured based on standard protocols [17] and their Body Mass Indexes (BMI) were calculated as the weight $(\mathrm{kg})$ divided by the square of height $(\mathrm{m} 2)$. Body Mass Indexes categories were classified based on the cut-off values recommended by Iran's National Institute of Health [18] as obese, BMI $\geq 30$; overweight, 25.0< $\mathrm{BMl}<29.9$; normal weight, 18.5< $\mathrm{BMl}<24.9$; and underweight, $\mathrm{BMl}<18.5 \mathrm{Kg} / \mathrm{m}^{2}$ [17]. Waist Circumference cut-offs for the Iranian adult population was considered as follows: $W C \geq 95 \mathrm{~cm}$ (high risk for Cardio-Vascular Disease (CVD) events), WC $\geq 90 \mathrm{~cm}$ (at risk for CVD risk factors), and WC $<90$ (no risk for CVD).

The research team developed the preliminary questionnaire based on the literature review. The questionnaire (IMB model) was reviewed afterwards by an expert panel $(n=15)$ and the participant population ( $n=10)$ for understanding, readability, and content validity. Assessment of reliability was done by a test-retest method and was confirmed by a Cronbach's alpha value greater than 0.8 . The items with Content Validity Ratio (CVR) $>0.49$ and Content Validity Index $(\mathrm{CVI})>0.79$ were considered appropriate.

Twelve items were used to assess the aspects of information (0-32 score), 10 items for personal motivation (10-50 score), 9 items for social motivation (9-45 score), 8 items for self-efficacy (8-40 score), and 10 items for self-regulation (10-50 score). Higher scores reflected higher amounts of IMB components [19]. The subjects were classified into three categories (low, middle, and high) based on tertiles of IMB components. Higher scores reflected higher amounts of accurate information, total motivation, and behavioral skills.

Dietary assessment was done using a validated semi-quantitative FFQ which was completed through face-to-face personal interviews. Food Frequency Questionnaire included 168 items adopted from Willett's format [20]. The subjects were asked to report their consumption of different food items on a daily, weekly, or monthly basis. The Food Frequency Questionnaire was validated for the study population and the validation study showed that those correlation coefficients between FFQ and 24 hour dietary recalls ranged from 0.32 to 0.72 , and were not substantially different between genders [21]. Food Frequency Questionnaire food items were grouped as follows: bread \& cereals; beans; vegetables; fruits; milk \& dairy products; red \& processed meat; fats \& oils; sugar; and salty foods.

Statistical analysis was done with the SPSS ${ }^{\circledR}$ Software version 20.0 (IBM, USA, 2011) [22] using Analysis of Variance (ANOVA) with following Tukey post-hoc test and bivariate correlation to test the 
association of SES and IMB components with food group consumption. $p$-values less than 0.05 were considered statistically significant.

The interrelationships among SES and IMB scores and consumption of healthy foods (bread \& cereals; beans; vegetables; fruits; milk \& dairy products) and unhealthy foods (red \& processed meat; fats \& oils; sugar; salty foods) were examined simultaneously through SEM analysis. The structure equation modeling used in this study is an extension of the General Linear Model that enables a researcher to test a set of regression equations simultaneously. It is a causal modeling technique, which depicts theorized, directional relationships among a set of observed and latent variables. We used the SPSS ${ }^{\circledR}$ Software (version 21.0) SPSS ${ }^{\circledR}$ Software AMOSTM (version 23.0) for SEM. Chi-Square test, Root Mean Square Error of Approximation (RMSEA), and Comparative Fit Index (CFI) were applied as statistics to test the model's fitness. Root Mean Square Error of Approximation $\leq 0.08$ and CFI values close to 0.95 were considered as an acceptable fit between the proposed model and the data set $[23,24]$. Associations between the research variables were measured using the regression coefficients ( $B$ ) at $p$-values below 0.05 [25], and the presented model parameters were standardized. Indirect associations were calculated as the product of the standardized regression coefficients of the pathway components [26]; this product was then divided by the product of the standard errors from the pathway components to assess the significance of the indirect effects. The modification index Lagrange Multiplier was applied to obtain a better-fitting model for each possible path that can be added to the model, if and when making the modification was theoretically consistent with the research goals.

\section{RES U LTS}

Differences in food group consumption based on the participants' socio-economic characteristics are shown in Table 1. Most women were married (80.6\%) and housewives (59.9\%), and differences in their dietary intake based on age, marital status, and employment status were not significant. The women living in northern Tehran had higher consumptions of red \& processed meat; in the eastern region, they showed higher milk \& dairy products intake; and in the southern region, a higher consumption of fat \& oils was found. Those living in the western part of the city had lower consumption of vegetables when compared to the other regions $(p<0.05)$.

The intake of red \& processed meat was found at its lowest level among less educated women, while more educated subjects consumed more fats \& oils $(p<0.05)$, as well as more bread \& cereals $(p<0.05)$. Women whose husbands were unemployed consumed less fruits and more sugar than those whose husbands had skilled occupations, and the retirees had the highest fruit consumption. Employees also consumed less bread \& cereals and salty foods than other groups. In households with lower total expenditures, the consumption of fats $\&$ oils was higher.

According to the information on Table 2, underweight women had the lowest consumption of vegetables and milk \& dairy products, while no significant difference was observed in the consumption of the food groups based on the women's WC. The consumption of milk \& dairy products, fats \& oils, sugar, and salty foods was higher among women with lower information scores. Women with lower attitude scores consumed less fruits and milk, and more sugar and salty foods, while high-attitude-score women consumed more vegetables. Those with higher perceived social support consumed more milk $\&$ dairy products $(p<0.05)$, more fats $\&$ oils $(p<0.05)$, and less bread \& cereals $(p<0.05)$. Women with more self-efficacy had higher consumptions of milk \& dairy products and less salty foods. However, those with low self-efficacy reported less vegetables, fruits, and milk \& dairy intake, and higher sugar consumption. 
Table 1. Socio-economic of food group consumption based on socio-economic characteristics in adult women of Tehran, Iran, 2017

\begin{tabular}{|c|c|c|c|c|c|c|c|c|}
\hline $\begin{array}{l}\text { Socio-economic } \\
\text { characteristics }\end{array}$ & $\begin{array}{l}\text { Bread \& } \\
\text { cereals }\end{array}$ & Vegetables & Fruits & $\begin{array}{l}\text { Milk \& dairy } \\
\text { products }\end{array}$ & $\begin{array}{c}\text { Red \& } \\
\text { processed } \\
\text { meat }\end{array}$ & Fats \& oils & Sugar & $\begin{array}{l}\text { Salty } \\
\text { foods }\end{array}$ \\
\hline & $\mathrm{M}(\mathrm{SE})$ & $\mathrm{M}(\mathrm{SE})$ & $\mathrm{M}(\mathrm{SE})$ & $\mathrm{M}(\mathrm{SE})$ & $\mathrm{M}(\mathrm{SE})$ & $\mathrm{M}(\mathrm{SE})$ & $\mathrm{M}(\mathrm{SE})$ & $\mathrm{M}(\mathrm{SE})$ \\
\hline \multicolumn{9}{|l|}{ Region } \\
\hline \multirow[t]{2}{*}{ North $(n=49)$} & 384.41 & 510.55 & 467.37 & 426.07 & 161.43 & 35.71 & 85.35 & 11.71 \\
\hline & (36.14) & $(30.67)$ & (29.75) & (33.63) & $(11.74)^{*}$ & (3.17) & (11.34) & $(2.44)$ \\
\hline \multirow[t]{2}{*}{ South $(n=48)$} & 434.56 & 558.75 & 418.76 & 499.44 & 152.94 & 46.06 & 84.94 & 22.85 \\
\hline & (30.04) & (33.35) & (31.44) & (34.55) & (11.06) & $(3.75)^{*}$ & (10.16) & $(3.87)^{*}$ \\
\hline \multirow[t]{2}{*}{ East $(n=49)$} & 425.98 & 581.80 & 438.97 & 514.50 & 145.11 & 34.13 & 62.95 & 12.77 \\
\hline & (34.18) & (29.63) & $(31.83)$ & $(40.91)^{*}$ & $(8.74)$ & $(2.72)$ & $(6.82)$ & $(2.15)$ \\
\hline \multirow[t]{2}{*}{ West $(n=52)$} & 386.47 & 461.10 & 425.18 & 384.93 & 134.72 & 29.83 & 64.46 & 9.91 \\
\hline & (25.41) & $(25.60)^{*}$ & $(25.71)$ & (30.71) & $(8.50)$ & $(2.49)$ & (6.65) & $(2.36)$ \\
\hline \multirow[t]{2}{*}{ Center $(n=49)$} & 461.56 & 549.18 & 410.77 & 405.43 & 126.75 & 35.44 & 70.61 & 8.94 \\
\hline & (33.48) & $(36.72)$ & $(32.60)$ & $(33.34)$ & $(10.04)$ & (3.06) & (10.09) & (1.78) \\
\hline \multicolumn{9}{|l|}{ Education } \\
\hline \multirow[t]{2}{*}{ Illiterate/primary $(n=16)$} & 395.09 & 527.39 & 498.88 & 548.89 & 113.59 & 38.70 & 79.92 & 15.52 \\
\hline & $(58.49)$ & $(55.47)$ & $(58.70)$ & $(71.94)$ & $(13.50)^{*}$ & (4.39) & $(15.37)$ & $(5.66)$ \\
\hline \multirow[t]{2}{*}{ Secondary $(n=105)$} & 455.29 & 551.62 & 416.00 & 458.05 & 136.95 & 40.85 & 76.25 & 13.24 \\
\hline & $(22.23)^{*}$ & $(21.82)$ & $(20.87)$ & (26.73) & $(6.63)$ & $(2.34)^{*}$ & $(6.43)$ & $(1.67)$ \\
\hline \multirow[t]{2}{*}{ Higher $(n=126)$} & 390.11 & 515.24 & 437.16 & 421.16 & 153.81 & 31.85 & 70.40 & 12.78 \\
\hline & $(19.44)$ & (19.73) & $(18.44)$ & (19.13) & $(6.62)$ & $(1.77)$ & $(5.71)$ & $(1.76)$ \\
\hline \multicolumn{9}{|l|}{ Spouse education } \\
\hline \multirow[t]{2}{*}{ Illiterate/primary $(n=12)$} & 414.59 & 488.03 & 383.05 & 479.15 & 119.26 & 35.68 & 92.82 & 15.75 \\
\hline & (54.19) & (63.98) & $(61.79)$ & $(83.51)$ & (9.84) & (5.69) & (19.90) & (6.96) \\
\hline \multirow[t]{2}{*}{ Secondary $(n=94)$} & 440.91 & 555.04 & 424.11 & 489.69 & 148.45 & 41.13 & 73.20 & 16.82 \\
\hline & $(24.40)$ & $(22.20)$ & (21.34) & $(27.52)$ & $(7.76)$ & $(2.24)^{\star}$ & $(61.76)$ & $(2.31)^{*}$ \\
\hline \multirow[t]{2}{*}{ Higher $(n=93)$} & 413.09 & 522.77 & 459.93 & 419.58 & 144.85 & 32.65 & 66.31 & 11.01 \\
\hline & (23.69) & (22.71) & $(22.48)$ & (22.96) & (7.85) & $(2.37)$ & $(6.56)$ & (1.67) \\
\hline \multicolumn{9}{|l|}{ Spouse occupation } \\
\hline \multirow[t]{2}{*}{ Unemployed $(n=7)$} & 580.01 & 468.72 & 296.70 & 491.89 & 131.30 & 35.50 & 139.17 & 23.19 \\
\hline & (148.41) & $(63.42)$ & $(61.08)^{*}$ & (101.09) & (44.39) & (3.77) & $(36.80)^{*}$ & $(10.50)$ \\
\hline \multirow[t]{2}{*}{ Worker $(n=24)$} & 398.09 & 571.54 & 457.77 & 515.36 & 125.05 & 41.55 & 65.60 & 17.71 \\
\hline & $(32.24)$ & $(46.84)$ & $(43.76)$ & $(60.57)$ & $(12.00)$ & $(4.60)$ & $(8.04)$ & $(4.48)$ \\
\hline \multirow[t]{2}{*}{ Employed (n=77) } & 387.37 & 519.56 & 454.68 & 418.95 & 139.63 & 33.22 & 59.07 & 8.42 \\
\hline & $(23.76)^{*}$ & $(24.82)$ & $(24.52)$ & $(26.89)$ & $(8.19)$ & $(2.68)$ & $(6.30)$ & $(1.32)^{*}$ \\
\hline \multirow[t]{2}{*}{ Retired $(n=6)$} & 388.65 & 570.15 & 594.36 & 364.71 & 138.51 & 36.96 & 55.36 & 7.89 \\
\hline & (65.54) & (108.10) & $(80.23)^{*}$ & (107.07) & (19.35) & $(9.22)$ & (12.11) & $(4.25)$ \\
\hline \multirow[t]{2}{*}{ Freelancer $(\mathrm{n}=85)$} & 459.58 & 543.80 & 421.96 & 476.97 & 157.11 & 38.89 & 79.20 & 17.78 \\
\hline & $(26.41)$ & $(23.40)$ & $(22.82)$ & $(26.39)$ & $(8.24)$ & $(2.40)$ & $(7.51)$ & $(2.58)$ \\
\hline \multicolumn{9}{|l|}{ Total expenditure } \\
\hline Low $(n=94)$ & 417.49 & 546.42 & 406.35 & 440.69 & 145.42 & 39.81 & 79.67 & 14.98 \\
\hline & $(23.48)$ & $(23.01)$ & $(21.85)$ & $(24.79)$ & $(6.88)$ & $(2.43)^{*}$ & (7.03) & $(1.88)$ \\
\hline Middle $(n=78)$ & 439.92 & 515.18 & 456.25 & 450.89 & 142.44 & 36.11 & 71.44 & 12.63 \\
\hline & $(25.63)$ & $(23.58)$ & $(22.47)$ & $(29.39)$ & $(7.63)$ & $(2.51)$ & $(6.50)$ & $(2.43)$ \\
\hline High $(n=75)$ & 396.31 & 529.76 & 439.52 & 444.66 & 143.97 & 31.50 & 67.93 & 11.42 \\
\hline & $(25.41)$ & $(27.30)$ & $(25.91)$ & $(28.49)$ & $(9.32)$ & $(2.17)$ & $(7.74)$ & $(1.89)$ \\
\hline T & 418.14 & 531.49 & 432.18 & 445.12 & $144 . .04$ & 36.12 & 73.51 & 13.16 \\
\hline Jutar (11-24) & (14.30) & $(14.14)$ & (13.48) & (15.75) & (4.53) & (1.40) & (4.11) & $(1.20)$ \\
\hline
\end{tabular}

Note: "Significant difference with other groups using ANOVA $(p<0.05)$.

M: Mean; SE: Standard Error. 
Table 2. Socio-economic of food group consumption based on anthropometrics characteristics and Information, Motivation, and Behavioral Skills Model scores in adult women of Tehran, Iran, 2017.

\begin{tabular}{|c|c|c|c|c|c|c|c|c|}
\hline $\begin{array}{l}\text { Anthropometric and IMB } \\
\text { groups }\end{array}$ & $\begin{array}{l}\text { Bread \& } \\
\text { cereals }\end{array}$ & Vegetables & Fruits & $\begin{array}{l}\text { Milk \& dairy } \\
\text { products }\end{array}$ & $\begin{array}{c}\text { Red \& } \\
\text { processed } \\
\text { meat }\end{array}$ & Fats \& oils & Sugar & $\begin{array}{l}\text { Salty } \\
\text { foods }\end{array}$ \\
\hline & $\mathrm{M}(\mathrm{SE})$ & $\mathrm{M}(\mathrm{SE})$ & $\mathrm{M}(\mathrm{SE})$ & $\mathrm{M}(\mathrm{SE})$ & $\mathrm{M}(\mathrm{SE})$ & $\mathrm{M}(\mathrm{SE})$ & $\mathrm{M}(\mathrm{SE})$ & $\mathrm{M}(\mathrm{SE})$ \\
\hline \multicolumn{9}{|l|}{ BMI status } \\
\hline Underweight (n=12) & $\begin{array}{l}402.75 \\
(64.13)\end{array}$ & $\begin{array}{c}335.71 \\
(52.65)^{*}\end{array}$ & $\begin{array}{l}397.70 \\
(65.23)\end{array}$ & $\begin{array}{c}260.05 \\
(64.46)^{*}\end{array}$ & $\begin{array}{l}120.60 \\
(13.25)\end{array}$ & $\begin{array}{l}40.78 \\
(6.15)\end{array}$ & $\begin{array}{c}81.78 \\
(14.67)\end{array}$ & $\begin{array}{c}5.71 \\
(1.46)\end{array}$ \\
\hline Normal weight $(n=97)$ & $\begin{array}{l}428.27 \\
(23.82)\end{array}$ & $\begin{array}{l}495.80 \\
(21.97)\end{array}$ & $\begin{array}{l}410.60 \\
(19.20)\end{array}$ & $\begin{array}{l}429.06 \\
(23.66)\end{array}$ & $\begin{array}{l}144.51 \\
(7.17)\end{array}$ & $\begin{array}{l}36.72 \\
(2.28)\end{array}$ & $\begin{array}{l}68.74 \\
(6.63)\end{array}$ & $\begin{array}{l}14.55 \\
(2.20)\end{array}$ \\
\hline Overweight $(n=85)$ & $\begin{array}{l}395.83 \\
(20.77)\end{array}$ & $\begin{array}{l}552.49 \\
(23.69)\end{array}$ & $\begin{array}{l}459.91 \\
(25.77)\end{array}$ & $\begin{array}{l}459.66 \\
(25.63)\end{array}$ & $\begin{array}{l}142.87 \\
(7.51)\end{array}$ & $\begin{array}{l}35.10 \\
(2.51)\end{array}$ & $\begin{array}{l}76.48 \\
(7.07)\end{array}$ & $\begin{array}{l}12.10 \\
(1.99)\end{array}$ \\
\hline Obese $(n=53)$ & $\begin{array}{l}438.89 \\
(35.31)\end{array}$ & $\begin{array}{l}607.47 \\
(29.20)\end{array}$ & $\begin{array}{l}439.08 \\
(28.01)\end{array}$ & $\begin{array}{l}497.89 \\
(38.20)\end{array}$ & $\begin{array}{l}150.36 \\
(10.99)\end{array}$ & $\begin{array}{l}34.16 \\
(1.40)\end{array}$ & $\begin{array}{l}75.58 \\
(9.08)\end{array}$ & $\begin{array}{l}12.55 \\
(2.10)\end{array}$ \\
\hline \multicolumn{9}{|l|}{ Waist circumferences } \\
\hline Normal $(n=159)$ & $\begin{array}{l}416.51 \\
(17.37)\end{array}$ & $\begin{array}{l}514.67 \\
(17.96)\end{array}$ & $\begin{array}{l}429.93 \\
(16.11)\end{array}$ & $\begin{array}{l}442.46 \\
(19.63)\end{array}$ & $\begin{array}{l}144.60 \\
(5.29)\end{array}$ & $\begin{array}{l}36.92 \\
(1.80)\end{array}$ & $\begin{array}{l}74.41 \\
(5.10)\end{array}$ & $\begin{array}{l}14.16 \\
(1.52)\end{array}$ \\
\hline At risk $(n=37)$ & $\begin{array}{l}434.28 \\
(38.46)\end{array}$ & $\begin{array}{l}533.72 \\
(36.45)\end{array}$ & $\begin{array}{l}416.01 \\
(35.56)\end{array}$ & $\begin{array}{l}415.15 \\
(35.26)\end{array}$ & $\begin{array}{l}138.63 \\
(12.45)\end{array}$ & $\begin{array}{l}32.67 \\
(2.80)\end{array}$ & $\begin{array}{l}69.81 \\
(9.51)\end{array}$ & $\begin{array}{l}10.07 \\
(2.37)\end{array}$ \\
\hline High risk $(n=51)$ & $\begin{array}{l}411.51 \\
(33.45)\end{array}$ & $\begin{array}{l}582.32 \\
(28.68)\end{array}$ & $\begin{array}{l}450.91 \\
(33.18)\end{array}$ & $\begin{array}{l}475.13 \\
(37.84)\end{array}$ & $\begin{array}{l}146.20 \\
(11.46)\end{array}$ & $\begin{array}{l}36.11 \\
(3.19)\end{array}$ & $\begin{array}{l}73.36 \\
(9.88)\end{array}$ & $\begin{array}{l}12.25 \\
(2.87)\end{array}$ \\
\hline \multicolumn{9}{|l|}{ Information } \\
\hline $\operatorname{Low}(n=80)$ & $\begin{array}{l}412.33 \\
(25.86)\end{array}$ & $\begin{array}{l}536.61 \\
(23.52)\end{array}$ & $\begin{array}{l}433.23 \\
(23.34)\end{array}$ & $\begin{array}{c}497.92 \\
(29.28)^{\star}\end{array}$ & $\begin{array}{c}155.38 \\
(8.61)\end{array}$ & $\begin{array}{c}41.13 \\
(2.76)^{*}\end{array}$ & $\begin{array}{l}80.65 \\
(7.89)\end{array}$ & $\begin{array}{c}18.73 \\
(2.73)^{*}\end{array}$ \\
\hline Middle $(n=92)$ & $\begin{array}{l}444.43 \\
(24.42)\end{array}$ & $\begin{array}{l}549.04 \\
(24.56)\end{array}$ & $\begin{array}{l}442.51 \\
(22.85)\end{array}$ & $\begin{array}{l}424.36 \\
(24.10)\end{array}$ & $\begin{array}{l}134.10 \\
(6.89)\end{array}$ & $\begin{array}{l}36.11 \\
(2.34)\end{array}$ & $\begin{array}{l}73.68 \\
(6.81)\end{array}$ & $\begin{array}{l}12.30 \\
(1.84)\end{array}$ \\
\hline High $(n=75)$ & $\begin{array}{l}392.09 \\
(23.57)\end{array}$ & $\begin{array}{l}504.51 \\
(25.17)\end{array}$ & $\begin{array}{l}418.39 \\
(24.04)\end{array}$ & $\begin{array}{l}414.25 \\
(27.37)\end{array}$ & $\begin{array}{c}143.03 \\
(8.09)\end{array}$ & $\begin{array}{l}30.79 \\
(1.92)\end{array}$ & $\begin{array}{l}65.66 \\
(6.49)\end{array}$ & $\begin{array}{c}8.27 \\
(1.11)\end{array}$ \\
\hline \multicolumn{9}{|l|}{ Attitude } \\
\hline $\operatorname{Low}(n=82)$ & $\begin{array}{l}449.64 \\
(29.92)\end{array}$ & $\begin{array}{l}509.30 \\
(24.62)\end{array}$ & $\begin{array}{c}367.68 \\
(21.54)^{*}\end{array}$ & $\begin{array}{c}390.82 \\
(23.77)^{*}\end{array}$ & $\begin{array}{c}147.21 \\
(7.59)\end{array}$ & $\begin{array}{l}38.91 \\
(2.41)\end{array}$ & $\begin{array}{c}95.63 \\
(8.28)^{*}\end{array}$ & $\begin{array}{c}16.80 \\
(2.13)^{*}\end{array}$ \\
\hline Middle $(n=76)$ & $\begin{array}{l}402.13 \\
(22.96)\end{array}$ & $\begin{array}{l}500.30 \\
(25.02)\end{array}$ & $\begin{array}{l}453.32 \\
(23.10)\end{array}$ & $\begin{array}{l}472.13 \\
(29.96)\end{array}$ & $\begin{array}{c}153.99 \\
(8.76)\end{array}$ & $\begin{array}{l}36.14 \\
(2.83)\end{array}$ & $\begin{array}{l}71.33 \\
(7.27)\end{array}$ & $\begin{array}{l}13.69 \\
(2.03)\end{array}$ \\
\hline High $(n=89)$ & $\begin{array}{l}402.79 \\
(20.67)\end{array}$ & $\begin{array}{c}578.58 \\
(23.27)^{\star}\end{array}$ & $\begin{array}{l}473.56 \\
(23.03)\end{array}$ & $\begin{array}{l}472.08 \\
(27.29)\end{array}$ & $\begin{array}{c}132.62 \\
(7.21)\end{array}$ & $\begin{array}{l}33.53 \\
(2.06)\end{array}$ & $\begin{array}{l}54.98 \\
(5.02)\end{array}$ & $\begin{array}{c}9.35 \\
(1.99)\end{array}$ \\
\hline \multicolumn{9}{|l|}{ Social support } \\
\hline $\operatorname{Low}(n=81)$ & $\begin{array}{c}464.54 \\
(28.11)^{*}\end{array}$ & $\begin{array}{l}536.72 \\
(24.34)\end{array}$ & $\begin{array}{l}417.80 \\
(26.29)\end{array}$ & $\begin{array}{l}416.19 \\
(26.19)\end{array}$ & $\begin{array}{l}142.51 \\
(8.66)\end{array}$ & $\begin{array}{l}34.58 \\
(2.29\end{array}$ & $\begin{array}{l}75.75 \\
(8.35)\end{array}$ & $\begin{array}{l}13.12 \\
(1.63)\end{array}$ \\
\hline Middle $(n=94)$ & $\begin{array}{l}381.34 \\
(20.21)\end{array}$ & $\begin{array}{l}512.90 \\
(22.70)\end{array}$ & $\begin{array}{l}433.70 \\
(20.44)\end{array}$ & $\begin{array}{l}423.34 \\
(22.69)\end{array}$ & $\begin{array}{c}139.01 \\
(6.70)\end{array}$ & $\begin{array}{l}31.86 \\
(1.74)\end{array}$ & $\begin{array}{l}66.38 \\
(4.93)\end{array}$ & $\begin{array}{l}12.00 \\
(1.95)\end{array}$ \\
\hline High $(n=72)$ & $\begin{array}{l}413.10 \\
(25.89)\end{array}$ & $\begin{array}{l}549.89 \\
(27.06)\end{array}$ & $\begin{array}{l}446.37 \\
(23.74)\end{array}$ & $\begin{array}{c}506.08 \\
(33.51)^{\star}\end{array}$ & $\begin{array}{l}152.31 \\
(80.40)\end{array}$ & $\begin{array}{c}43.40 \\
(3.20)^{\star}\end{array}$ & $\begin{array}{l}80.28 \\
(8.30)\end{array}$ & $\begin{array}{l}14.70 \\
(2.66)\end{array}$ \\
\hline \multicolumn{9}{|l|}{ Self-efficacy } \\
\hline Low $(n=95)$ & $\begin{array}{l}434.97 \\
(25.42)\end{array}$ & $\begin{array}{c}476.24 \\
(21.39)^{*}\end{array}$ & $\begin{array}{c}382.89 \\
(21.39)^{*}\end{array}$ & $\begin{array}{l}401.24 \\
(21.47)\end{array}$ & $\begin{array}{c}148.99 \\
(7.76)\end{array}$ & $\begin{array}{l}37.32 \\
(2.22)\end{array}$ & $\begin{array}{c}86.56 \\
(7.02)^{*}\end{array}$ & $\begin{array}{l}15.21 \\
(1.77)\end{array}$ \\
\hline Middle $(n=65)$ & $\begin{array}{l}434.66 \\
(27.09)\end{array}$ & $\begin{array}{l}553.45 \\
(26.88)\end{array}$ & $\begin{array}{l}458.89 \\
(24.77)\end{array}$ & $\begin{array}{l}415.07 \\
(31.85)\end{array}$ & $\begin{array}{c}147.34 \\
(8.79)\end{array}$ & $\begin{array}{l}36.01 \\
(2.95)\end{array}$ & $\begin{array}{l}70.20 \\
(7.91)\end{array}$ & $\begin{array}{l}15.62 \\
(3.06)\end{array}$ \\
\hline High $(n=87)$ & $\begin{array}{l}387.43 \\
(21.53)\end{array}$ & $\begin{array}{l}575.42 \\
(24.70)\end{array}$ & $\begin{array}{l}466.04 \\
(23.21)\end{array}$ & $\begin{array}{c}515.47 \\
(28.45)^{*}\end{array}$ & $\begin{array}{c}136.17 \\
(7.12)\end{array}$ & $\begin{array}{l}34.89 \\
(2.26)\end{array}$ & $\begin{array}{l}61.71 \\
(6.29)\end{array}$ & $\begin{array}{c}9.07 \\
(1.53)^{*}\end{array}$ \\
\hline \multicolumn{9}{|l|}{ Self-regulation } \\
\hline $\operatorname{Low}(n=80)$ & $\begin{array}{l}472.15 \\
(28.82)^{*}\end{array}$ & $\begin{array}{l}508.26 \\
(24.77)\end{array}$ & $\begin{array}{c}384.59 \\
(24.71)^{*}\end{array}$ & $\begin{array}{l}414.71 \\
(25.24)\end{array}$ & $\begin{array}{l}151.58 \\
(8.11)\end{array}$ & $\begin{array}{l}38.74 \\
(2.78)\end{array}$ & $\begin{array}{c}86.30 \\
(7.65)^{\star}\end{array}$ & $\begin{array}{c}19.47 \\
(2.73)^{*}\end{array}$ \\
\hline Middle $(n=87)$ & $\begin{array}{l}414.56 \\
(23.13)\end{array}$ & $\begin{array}{l}553.17 \\
(24.62)\end{array}$ & $\begin{array}{l}457.54 \\
(21.46)\end{array}$ & $\begin{array}{l}479.02 \\
(26.99)\end{array}$ & $\begin{array}{l}151.28 \\
(8.15)\end{array}$ & $\begin{array}{l}37.51 \\
(2.35)\end{array}$ & $\begin{array}{l}70.39 \\
(6.53)\end{array}$ & $\begin{array}{l}13.18 \\
(1.77)\end{array}$ \\
\hline High $(n=80)$ & $\begin{array}{l}368.03 \\
(20.79)\end{array}$ & $\begin{array}{l}531.15 \\
(24.03)\end{array}$ & $\begin{array}{l}452.18 \\
(23.43)\end{array}$ & $\begin{array}{l}438.65 \\
(29.26)\end{array}$ & $\begin{array}{l}128.62 \\
(6.96)^{*}\end{array}$ & $\begin{array}{l}31.98 \\
(2.03)\end{array}$ & $\begin{array}{l}64.10 \\
(7.06)\end{array}$ & $\begin{array}{c}6.82 \\
(1.25)\end{array}$ \\
\hline
\end{tabular}

Note: * Significant difference between groups using ANOVA $(p<0.05)$.

IMB: Information, Motivation, and Behavioral Skills Model; M: Mean; SE: Standard Error. 
Those with high self-regulation scores reported less consumption of red \& processed meat, while low-score women reported higher sugar, salty foods, bread \& cereals consumption, as well as less fruit intake than other groups.

The number of rooms in the house as an indicator of SES negatively correlated with the consumption of vegetables, milk \& dairy products, fats \& oils, sugar, and salty foods. In addition, consumption of red \& processed meat was negatively associated with family size and positively associated with living area per person. In turn, the living area per person had a negative relationship with the intake of bread \& cereals. Vegetable consumption had positive correlation with BMI and WC; however, only the BMI had a positive correlation with milk \& dairy products intake in women. Total expenditure was negatively related to the intake of fats and oils. The majority of IMB components were positively correlated with the consumption of fruits and milk \& dairy products, and negatively correlated with the consumption of fats \& oils, sugar, and salty foods. Moreover, a negative relationship was found between bread \& cereals intake, on one hand, and both self-efficacy and self-regulation, on the other. The detailed results of the correlation tests are shown in Table 3.

The data provided in Table 4 and Figure 1 reveal that the IMB components were the most important factors affecting the consumption of unhealthy foods (red \& processed meat, fats \& oils,

Table 3. Association among food group consumption, socio-economic characteristics, and Information, Motivation, and Behavioral Skills model scores in adult women of Tehran, Iran- 2017.

\begin{tabular}{|c|c|c|c|c|c|c|c|c|}
\hline $\begin{array}{l}\text { Socio-economic } \\
\text { characteristics }\end{array}$ & $\begin{array}{l}\text { Bread \& } \\
\text { cereals }\end{array}$ & Vegetables & Fruits & $\begin{array}{l}\text { Milk \& dairy } \\
\text { products }\end{array}$ & $\begin{array}{c}\text { Red \& } \\
\text { processed } \\
\text { meat }\end{array}$ & Fats \& oils & Sugar & Salty foods \\
\hline & $\begin{array}{l}\text { Pearson r } \\
\text { (p-value) }\end{array}$ & $\begin{array}{l}\text { Pearson r } \\
\text { (p-value) }\end{array}$ & $\begin{array}{l}\text { Pearson r } \\
\text { (p-value) }\end{array}$ & $\begin{array}{l}\text { Pearson r } \\
\text { (p-value) }\end{array}$ & $\begin{array}{l}\text { Pearson r } \\
\text { (p-value) }\end{array}$ & $\begin{array}{l}\text { Pearson } r \\
\text { (p-value) }\end{array}$ & $\begin{array}{l}\text { Pearson } r \\
\text { (p-value) }\end{array}$ & $\begin{array}{l}\text { Pearson r } \\
\text { (p-value) }\end{array}$ \\
\hline \multirow[t]{2}{*}{ Age (years) } & -0.044 & 0.083 & 0.075 & 0.024 & -0.058 & -0.053 & -0.009 & -0.050 \\
\hline & $(0.487)$ & $(0.194)$ & $(0.241)$ & $(0.708)$ & $(0.362)$ & $(0.403)$ & $(0.887)$ & $(0.438)$ \\
\hline \multirow[t]{2}{*}{ Family size } & 0.104 & 0.003 & -0.002 & 0.003 & -0.206 & -0.046 & -0.045 & -0.101 \\
\hline & $(0.103)$ & $(0.957)$ & $(0.720)$ & $(0.965)$ & $(0.001)^{* *}$ & $(0.473)$ & $(0.486)$ & $(0.115)$ \\
\hline \multirow[t]{2}{*}{ Number of room } & -0.156 & -0.152 & 0.068 & -0.137 & -0.041 & -0.160 & -0.134 & -0.218 \\
\hline & $(0.014)$ & $(0.017)^{*}$ & $(0.287)$ & $(0.031)^{*}$ & $(0.519)$ & $(0.012)^{*}$ & $(-0.035)^{*}$ & $(0.001)^{* *}$ \\
\hline Total expenditure & -0.071 & -0.075 & 0.093 & -0.011 & 0.025 & -0.196 & -0.051 & -0.093 \\
\hline$\left(1000\right.$ Iranian Rials) ${ }^{* * *}$ & $(0.266)$ & $(0.242)$ & $(0.147)$ & $(0.861)$ & $(0.697)$ & $(0.002)^{* *}$ & $(0.428)$ & $(0.145)$ \\
\hline \multirow[t]{2}{*}{$\mathrm{BMI}\left(\mathrm{Kg} / \mathrm{m}^{2}\right)$} & 0.033 & 0.219 & 0.094 & 0.148 & 0.072 & -0.060 & 0.015 & 0.010 \\
\hline & $(0.602)$ & $(0.001)^{* *}$ & $(0.142)$ & $(0.020)^{*}$ & $(0.257)$ & $(0.350)$ & $(0.814)$ & $(0.876)$ \\
\hline \multirow{2}{*}{$\begin{array}{l}\text { Waist circumferences } \\
(\mathrm{cm})\end{array}$} & 0.010 & 0.206 & 0.115 & 0.097 & 0.037 & -0.044 & -0.002 & -0.02 \\
\hline & $(0.880)$ & $(0.001)^{* *}$ & $(0.070)$ & $(0.130)$ & $(0.567)$ & $(0.490)$ & $(0.980)$ & $(0.729)$ \\
\hline \multirow{2}{*}{$\begin{array}{l}\text { Living area per person } \\
\left(\mathrm{m}^{2} / \mathrm{p}\right)\end{array}$} & -0.148 & -0.055 & 0.071 & 0.005 & 0.132 & -0.055 & 0.017 & -0.049 \\
\hline & $(0.020)^{*}$ & $(0.388)$ & $(0.269)$ & $(0.938)$ & $(0.038)^{*}$ & $(0.388)$ & $(0.785)$ & $(0.446)$ \\
\hline \multirow[t]{2}{*}{ Information } & 0.015 & -0.047 & -0.002 & -0.148 & -0.020 & -0.230 & -0.093 & -0.210 \\
\hline & $(0.816)$ & $(0.466)$ & $(0.973)$ & $(0.020)^{*}$ & $(0.757)$ & $(0.000)^{*}$ & $(0.143)$ & $(0.001)^{* *}$ \\
\hline \multirow[t]{2}{*}{ Attitude } & -0.075 & 0.115 & 0.141 & 0.121 & -0.039 & -0.127 & -0.250 & -0.203 \\
\hline & $(0.240)$ & $(0.072)$ & $(0.027)^{*}$ & $(0.058)$ & $(0.545)$ & $(0.046)^{*}$ & $(0.000)^{* *}$ & $(0.001)^{* *}$ \\
\hline \multirow[t]{2}{*}{ Social-support } & -0.069 & 0.037 & 0.066 & 0.130 & 0.048 & 0.149 & 0.097 & 0.021 \\
\hline & $(0.279)$ & $(0.559)$ & $(0.299)$ & $(0.042)^{*}$ & $(0.454)$ & $(0.019)^{*}$ & $(0.128)$ & $(0.744)$ \\
\hline \multirow[t]{2}{*}{ Self-efficacy } & -0.168 & 0.110 & 0.147 & 0.154 & -0.108 & -0.131 & -0.230 & -0.215 \\
\hline & $(0.008)^{* *}$ & $(0.083)$ & $(0.021)^{*}$ & $(0.016)^{*}$ & $(0.090)$ & $(0.040)^{*}$ & $(0.000)^{* *}$ & $(0.001)^{* *}$ \\
\hline \multirow[t]{2}{*}{ Self-regulation } & -0.231 & 0.047 & 0.164 & 0.074 & -0.138 & -0.111 & -0.178 & -0.0264 \\
\hline & $(0.000)^{* *}$ & $(0.464)$ & $(0.010)^{*}$ & $(0.247)$ & $(0.030)^{*}$ & $(0.081)$ & $(0.005)^{* *}$ & $(0.000)^{* *}$ \\
\hline
\end{tabular}

Note: ${ }^{*}$ Correlation is significant at 0.05 level (2-tailed); ${ }^{* *}$ Correlation is significant at 0.01 level $\left(2\right.$-tailed); ${ }^{* * *} 4200$ Iranian Rials = 1 US Dollar. BMI: Body Mass Indexes. 
Table 4. Structural equation modeling analysis of the interrelationships of socio-economic status and Information, Motivation, and Behavioral Skills model components with healthy and unhealthy food group consumption in adult women in Tehran, Iran, 2017.

\begin{tabular}{|c|c|c|c|c|c|}
\hline Dependent & Independent & Standardized $\beta$ & $\mathrm{SE}^{*}$ & $C R^{* *}$ & $p$-value \\
\hline Healthy foods & $\mathrm{IMB}$ & 10.181 & 5.685 & 1.791 & .073 \\
\hline Unhealthy foods & IMB & -2.026 & 0.593 & -3.416 & $<0.0001$ \\
\hline Healthy foods & SES & 10.256 & 9.677 & 1.060 & 0.289 \\
\hline Unhealthy foods & SES & 0.267 & 0.906 & 0.295 & 0.768 \\
\hline Region & SES & 0.035 & 0.074 & 0.475 & 0.635 \\
\hline Education & SES & 0.041 & 0.033 & 1.242 & 0.214 \\
\hline Spouse education & SES & 0.826 & 0.128 & 6.466 & $<0.0001$ \\
\hline Spouse occupation & SES & 1.000 & & & \\
\hline Living area per person (m²/p) & SES & -1.061 & 0.800 & -1.327 & 0.0185 \\
\hline Family size & SES & -0.241 & 0.061 & -3.922 & $<0.0001$ \\
\hline Self-regulation & IMB & 2.027 & 0.312 & 6.487 & $<0.0001$ \\
\hline Self-efficacy & IMB & 1.435 & 0.221 & 6.486 & $<0.0001$ \\
\hline Social support & IMB & 0.863 & 0.212 & 4.074 & $<0.0001$ \\
\hline Attitude & IMB & 1.000 & & & \\
\hline Information & IMB & 0.247 & 0.089 & 2.782 & 0.005 \\
\hline Number of rooms & SES & -0.119 & 0.040 & -2.988 & 0.003 \\
\hline Total expenditure & SES & 80.286 & 85.709 & 2.631 & 0.009 \\
\hline Red \& processed meat & Unhealthy & 2.645 & 0.432 & 6.119 & $<0.0001$ \\
\hline Fats \& oils & Unhealthy & 1.000 & & & \\
\hline Sugar & Unhealthy & 2.351 & 0.356 & 6.598 & $<0.0001$ \\
\hline Salty foods & Unhealthy & .636 & 0.101 & 6.281 & $<0.0001$ \\
\hline Bread \& cereals & Healthy & 0.484 & 0.119 & 4.051 & $<0.0001$ \\
\hline Beans & Healthy & 0.095 & 0.022 & 4.256 & $<0.0001$ \\
\hline Vegetables & Healthy & 1.000 & & & \\
\hline Fruits & Healthy & 0.712 & 0.121 & 5.881 & $<0.0001$ \\
\hline Milk \& dairy products & Healthy & 0.866 & 0.143 & 6.059 & $<0.0001$ \\
\hline
\end{tabular}

Note: "Standard Error; ${ }^{* *}$ Composite Reliability.

IMB: Information, Motivation, and Behavioral Skills model; SES: Socio-Economic Status.

sugar, and salty foods). All components of the IMB model, including information, attitude, social support, self-efficacy, and self-regulation had a negative effect on the consumption of unhealthy food groups ( $t$ value $=-2.026, p<0.0001$ ). The socio-economic factors consisting of spouse education, family size, living area per person, number of rooms, and total expenditure did not affect the consumption of healthy and unhealthy foods. The model had relatively good fit with the data (RMSEA 0.063; CFI 0.824; Chi-Square 379.522, p<0.0001).

\section{S C U S S I O N}

The present study showed that food group consumption among Iranian adult women differed based on their SES and IMB components, including information, attitude, social support, self-efficacy, and self-regulation. The study focused on women because their lifestyle characteristics and food group consumption affect the whole household consumption, and they usually play a crucial role in food preparation and cooking.

We found that, as hypothesized, food group consumption was significantly related to the participants' SES, including district of living, educational and occupational levels of the husbands 


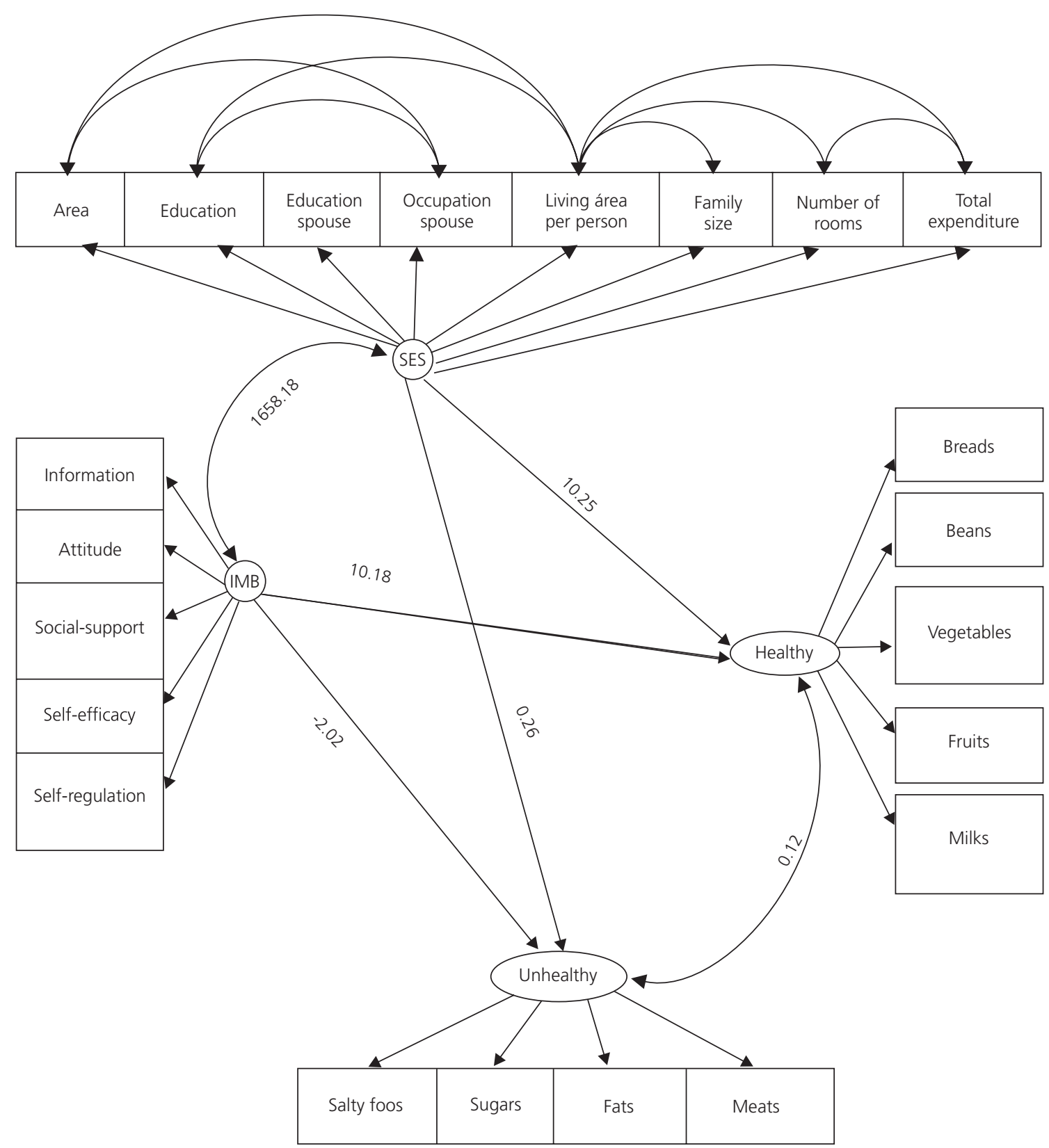

Figure 1. Structural equation modeling of relationship between socio-economic status, information-motivation and behavioral skills model componetnts, healthy and unhealthy food group consumption in adult women of Tehran, Iran, 2017.

Note: Root Mean Square Error Approximation 0.063 (90\% Confidence Interval: 0.054-0.072); Comparative Fit Index 0.824; Root Mean Square Residual 3059185.066; Goodness of Fit Index 0.878; Chi Square.

and wives, and household expenditure. Women with lower education and unemployed husbands tend to show a higher consumption of bread \& cereals, fats \& oils, and salty foods, as well as a lower consumption of red \& processed meat.

Moreover, low consumption of fruits and high consumption of sugar were seen in the households with unemployed men. Higher level of education could increase the families' ability to obtain healthy dietary information, promoting behaviors and beliefs in the field of food habits; also, 
the occupational level was found to effect on the household's purchasing power. Other studies have also shown that education is the strongest socio-economic determinant for healthy food consumption when compared to occupation and income $[5,27,28]$. We found that more rooms in the house had a negative effect on the consumption of vegetables, milk \& dairy products, fats \& oils, and salty foods. The findings from previous researchers defended that people in higher socio-economic classes have healthier diets. A healthy diet could be defined by a higher consumption of raw and cooked vegetables, fresh and dried fruits, cereals, and less consumption of red \& processed meat, sauces and dressings, fast foods, sweets, snacks, desserts, and sugars [3,29].

Studies of Abdollahi et al. [14] and Omidvar [30] and in Iran showed that raising the level of education increased the consumption of all food groups. Previous studies have also found that higher educational and occupational levels were associated with increased income and consumption of red \& processed meat, vegetables, fruits, eggs, sugar, and fast foods. However, Eshghinia et al. [31] and Rezazadeh et al. [32] recently showed significant increases in healthy dietary habits with progression in SES.

In the present study, underweight women had low vegetable and milk consumptions. In other studies, the higher consumption of vegetables is usually indicative of a healthy dietary pattern, which has been found to be associated with a normal BMI $[6,33,34]$. This can be attributed to the fact that lower SES is associated with lower consumption of vegetables and milk, which can also partly explain underweight issues in women.

Research results have shown that the IMB model's components have significantly affected the food group consumption. With the increase of information, attitude, social support, selfefficacy, and self-regulation, consumption of healthy foods increased and consumption of unhealthy foods decreased. Fisher and Fisher, who proposed the IMB model in 1992, suggest three required constructs (information, motivation, and behavioral skills) for improving a specific health behavior, as important individual predictors of behavioral change. Information, motivation, and behavioral skills as well as explicit relationships among these constructs are considered generalizable determinants of health behaviors [35,36]. In Great Britain and Denmark, the outcome of two cohort studies showed that dietary habits are influenced by economic capacity, dietary knowledge, attitudes, beliefs, and food preferences [37]. Women are generally more aware of healthy eating than men. Furthermore, women are more concerned about weight control and have a higher frequency of dieting than men. These gender-related behaviors are probably founded early in life, carried forward, and thus, may influence dietary patterns in adulthood [38]. Higher autonomous motivation, self-efficacy, and self-regulation skills have emerged as the best predictors of beneficial weight and physical activity outcomes; for weight control, positive body image and flexible eating restraint may additionally improve outcomes [39].

The IMB model was first used in this study as a conceptual framework for predictors of dietary behaviors in a group of women and it was shown that the constructs of this model were able to predict behavior in women well. In addition, socio-economic factors affecting the model were identified. The proposed model relies on limited structures and no complexity, it is not costly, and had its preventive role in nutritional behavior modification confirmed in Iranian socio-cultural contexts. The present study pointed to the IMB model's components as the most important factors that negatively affect the consumption of unhealthy foods (red \& processed meat, fats \& oils, sugar, and salty foods). In a study using the IMB model, Goodel et al. [40] found preliminary support for relationships between parental information, motivation, behavioral skills, and children's consumption of sweetened beverages. They also identified the complex interactions among the factors influencing 
the clients' consumption behaviors. Kelly et al. [41] showed the mediation of knowledge and personal motivation for both physical activity and fruit and vegetable intake through cognitive-behavioral skills. In addition, the paths leading to social support were significant.

Therefore, by promoting the constructs of the IMB model (information, attitude, social support, self-efficacy, and self-regulation), the consumption of unhealthy foods may be decreased. Moreover, besides unhealthy nutrition, the socioeconomic determinants, especially age, level of education, and expenditure, as well as self regulation, play an important role in excessive weight and central obesity among women [42].

The limitations of the current study included the use of a cross-sectional design that makes it difficult to identify the causal relationships among the variables. Nevertheless, conducting a largescale study could be accounted as a major strength of the present research. Other potential limitation of our study is the bias that can be derived from the over-reporting of consumption among those with higher levels of education. Also, this study was limited to the city of Tehran. Thus, the results could not be generalized to the whole country.

The Information, Motivation, and Behavioral Skills model has been extensively applied for over a decade to predict positive health behaviors in multiple populations. However, to the best of our knowledge, no published study has focused on improving the food group consumption based on the IMB model among women. In addition, our dietary measurement method has advantages; the applied FFQ has been previously validated and has proven to provide a valid estimate of habitual dietary consumption [21].

\section{CONCLUSION}

Structural equation modeling showed the direct effect of model components including information, attitude, and social support on behavioral skills (self-efficacy and self-regulation). Moreover, these components affected dietary behaviors (consumption of vegetables, fruits, oil, sugar, and salty foods) directly and indirectly by mediating behavioral skills. As nutrition educators frequently desire to alter behaviors, using the IMB model could be applicable for both targeting and assessment efforts. Researchers are able to see which foods make up a large portion of the women's diet. It requires more attention to the issue of theory-based education to promote healthy nutritional behaviors in women and their communities. The analysis of food consumption based on SES and the IMB model helps in the prevention of unhealthy diet patterns and improving healthy diet patterns, and thus represents a fundamental tool for better nutritional programming and policy making.

This research indicated that promoting healthy nutritional behaviors in women and the future design of intervention programs should be based on theory, while socio-economic characteristics should also be taken into account. The specific integration strategies delineated for each construct of the model can be utilized to design model-based interventions.

\section{ACKNOWLEDGEMENTS}

The authors would like to express their appreciation for the Deputy of Education, School of Public Health, Tehran University of Medical Sciences (Tehran, Iran). We would also like to acknowledge the women who participated in this research by completing questionnaires, as well as the social workers, community centers, and health houses affiliated with the Municipality of Tehran for their kind cooperation. 


\section{CONTRIBUTORS}

M MOHAMMADI-NASRABADI and R SADEGHI contributed in all phases of the study. A RAHIMI-FORUSHANI contributed in design, statistical analysis, manuscript preparation, and revision. D SHOJAEIZADE contributed in study conception, design, and definition of the intellectual content. F MOHAMMADI-NASRABADI contributed in design literature search, dietary data acquisition and analysis, manuscript preparation, and revision. A MONTAZERI contributed in design, data analysis, manuscript preparation, and revision.

\section{REFERENCES}

1. Ghassemi H, Harrison G, Mohammad K. An accelerated nutrition transition in Iran. Public Health Nutr. 2002;5(1A):149-55.

2. McCartney DM, Younger K, Kearney JM. Socio-economic differences in food group and nutrient intakes among young women in Ireland. $\mathrm{Br} J$ Nutr. 2013;110(11):2084-97. http://dx.doi.org/10.1017/S0 007114513001463

3. Hare-Bruun $\mathrm{H}$, Togo $\mathrm{P}$, Andersen LB, Heitmann BL. Adult food intake patterns are related to adult and childhood socioeconomic status. J Nutr. 2011;141(5):928-34. http://dx.doi.org/10.3945/jn.110.1334 13

4. Hulshof K, Brussaard J, Kruizinga A, Telman J, Löwik MR. Socio-economic status, dietary intake and 10 y trends: the Dutch National Food Consumption Survey. Eur J Clin Nutr. 2003;57(1):128-37.

5. Irala-Estevez JD, Groth M, Johansson L, Oltersdorf V, Prättälä R, Martinez-Gonzalez M. A systematic review of socio-economic differences in food habits in Europe: consumption of fruit and vegetables. Eur J Clin Nutr. 2000;54:709-14.

6. Sanchez-Villegas A, Martinez J, Prättälä R, Toledo E, Roos G, Martinez-Gonzalez M. A systematic review of socioeconomic differences in food habits in Europe: consumption of cheese and milk. Eur J Clin Nutr. 2003;57(8):917-29.

7. Groth MV, Fagt S, Brondsted L. Social determinants of dietary habits in Denmark. Eur J Clin Nutr. 2001;55:959-66.

8. Hupkens C, Knibbe R, Drop M. Social class differences in food consumption: the explanatory value of permissiveness and health and cost considerations. Eur J Public Health. 2000;10:108-13.

9. Darmon N, Drewnowski A. Does social class predict diet quality? Am J Clin Nutr. 2008;87:1107-17.

10. Giskes K, Turrell G, Patterson C, Newman B. Socioeconomic differences among Australian adults in consumption of fruit and vegetables and intakes of vitamins A, C and folate. J Hum Nutr Diet. 2002;15:375-85.

11. Esfahani FH, Ejtahed HS, Mirmiran P, Delshad H, Azizi F. Alterations in food group intakes and subsequent weight changes in adults: Tehran lipid and glucose study. Int J Endocrinol Metab. 2014;12(3):e17236. http:// dx.doi.org/10.5812/ijem.17236

12. Zeyninejad E, Omidvar N, Neyestani T, Houshiarrad A, Eshraghian MR, Stormer A. Development and validation of a food frequency questionnaire for assessing dietary calcium in children. Nutr Food Sci Res. 2015;2(1):34-45.

13. Bezerra IN, Goldman J, Hoy DGRK, Souza ADM, Chester DN, Martin CL, et al. Difference in adult food group intake by sex and age groups comparing Brazil and United States nationwide surveys. Nutr J. 2014;13:74-83. http://dx.doi.org/10.1186/1475-2891-13-74

14. Abdollahi M, Mohammadi-Nasrabadi F, Houshiarrad A, Ghaffarpur M, Ghodsi D, Kalantari N. Socio-economic differences in dietary intakes: the comprehensive study on household food consumption patterns and nutritional status of I.R. Iran. Nutr Food Sci Res. 2014;1(1):19-26.

15. Bian C, Xu S, Wang H, Li N, Wu J, Zhao Y, et al. A study on the application of the information-motivationbehavioral skills (IMB) model on rational drug use behavior among second-level hospital outpatients in Anhui, China. Plos One. 2015;10(8):1-11.

16. MacCallum RC, Widaman KF, Zhang S, Hong S. Sample size in factor analysis. Psychol Methods. 1999:4:84-99. 
17. National Institute of Health. Clinical guidelines on the identification, evaluation, and treatment of overweight and obesity in adults: the evidence report. Obes Res. 1998;6(S2):51S-179S. http://dx.doi. org/10.1002/j.1550-8528.1998.tb00690.x

18. Azizi F, Khalili D, Aghajani H, Esteghamati A, Hosseinpanah F, Delavari A, et al. Appropriate waist circumference cut-off points among Iranian adults: the first report of the Iranian National Committee of Obesity. Arch Iran Med. 2010;13(3):243-4.

19. Mohammadi-Nasrabadi M, Sadeghi R, Rahimiforushani A, Shojaeizadeh D, Montazeri A, MohammadiNasrabadi F. An educational intervention to promote healthy nutritional behavior in women: design and the framework. Tehran: Tehran University of Medical Sciences; 2018.

20. Willett WC. Nutritional epidemiology. New York: Oxford University Press; 1998.

21. Esfahani FH, Asghari G, Mirmiran P, Azizi F. Reproducibility and relative validity of food group intake in a food frequency questionnaire developed for the Tehran lipid and glucose study. J Epidemiol. 2010;20(2):150-8. http://dx.doi.org/10.2188/jea.JE20090083

22. International Business Machines Corporation. SPSS Statistics [software]. New York; 2011.

23. Byrne B. Structural equation modeling with AMOS. New Jersey: Lawrence Erlbaum Associates Inc.; 2001.

24. Hooper D, Coughlan JR, Mullen M. Structural equation modelling: guidelines for determining model fit. Electron J Bus Res Methods. 2008;6(1):53-60.

25. Schumacker RE, Lomax RG. A beginner's guide to structural equation modeling. New Jersey: LawrenceErlbaum Associates Inc.; 2004.

26. Kline RB. Principles and Practices of Structural Equation Modeling. 2nd ed., New York: The Guilford Press; 2005.

27. Kell KP, Judd SE, Pearson KE, Shikany JM, Fernández JR. Associations between socio-economic status and dietary patterns in US black and white adults. Br J Nutr. 2015;113(11):1792-9.

28. Moreira PA, Padrao PD. Educational and economic determinants of food intake in Portuguese adults: a cross-sectional survey. BMC Public Health. 2004;4:58.

29. Pechey R, Monsivais P. Socioeconomic inequalities in the healthiness of food choices: exploring the contributions of food expenditures. Prev Med. 2016;88:203-9.

30. Omidvar N. Psycho-social determinants of food choices and nutritional status of high school adolescents in Tehran. Iran: Tabriz University of Medical Sciences; 2002 [In Farsi].

31. Eshghinia S, Khodarahmi M, Bartimar R, Lashkarboluki F, Asadi J, Saneei P, et al. Socio-economic status versus dietary habits in women living in northeastern Iran. Iran J Nutr Sci Food Tech. 2014;9(1):21-9 [In Farsi].

32. Rezazadeh A, Rashidkhani B, Omidvar N. Association of major dietary patterns with socioeconomic and lifestyle factors of adult women living in Tehran, Iran. Nutr Food Sci Res. 2010;26(3):337-41.

33. Fokeena WB, Jamaluddin $R$, Khaza'ai $H$. Contribution of different food groups to the energy intake and weight status of adults: a cross-sectional study in a Malaysian Public University. Asian Clin Nutr. 2015;7(2):45-54.

34. Mozaffarian D, Hao T, Rimm EB, Willett WC, Hu FB. Changes in diet and lifestyle and long-term weight gain in women and men. N Engl J Med. 2011;346:2392-404.

35. Gao J, Wang J, Zhu Y, Yu J. Validation of an information-motivationbehavioral skills model of self-care among Chinese adults with type 2 diabetes. BMC Public Health. 2003;13:100.

36. Fisher WA, Fisher JD, Harman J. The information-motivation-behavioral skills model: a general social psychological approach to understanding and promoting health behavior. In: Suls J, Wallston KA, editors. Social Psychological Foundation of Health and Illness. Malden: Blackwell; 2003. p.82-106.

37. Turrell G. Socioeconomic differences in food preference and their influence on healthy food purchasing choices. J Hum Nutr Diet. 1998;11:135-49.

38. Wardle J, Haase AM, Steptoe A, Nillapun M, Jonwutiwes K, Bellisle F. Gender differences in food choice: the contribution of health beliefs and dieting. Ann Behav Med. 2004;27:107-16.

39. Teixeira PJ, Carraça EV, Marques MM, Rutter H, Oppert JM, Bourdeaudhuij ID, et al. Successful behavior change in obesity interventions in adults: a systematic review of self-regulation mediators. BMC Med. 2015;13:84. 
40. Goodell LS, Pierce MB, Amico KR, Ferris AM. Parental information, motivation, and behavioral skills correlate with child sweetened beverage consumption. J Nutr Educ Behav. 2012;44:240-5.

41. Kelly S, Melnyk BM, Belyea M. Predicting physical activity and fruit and vegetable intake in adolescents: a test of the Information, Motivation, Behavioral Skills model. Res Nurs Health. 2012;35(2):146-63. http://dx.doi. org/10.1002/nur.21462

42. Mohammadi-Nasrabadi M, Sadeghi R, Rahimiforushani A, Mohammadi-Nasrabadi F, Shojaeizadeh D, Montazeri A. Socioeconomic determinants of excess weight and central obesity among Iranian women: application of information, motivation, and behavioral skills model. J Educ Health Promot. 2019;8:75. http:// dx.doi.org/10.4103/jehp.jehp_427_18 